\title{
The Seeking Solutions Approach: Solving Challenging Business Problems with Local Open Innovation
}

\author{
Christophe Deutsch
}

\author{
"The world is becoming too fast, too complex," \\ and too networked for any company to have all \\ the answers inside.
}

\author{
Yochai Benkler \\ Law Professor and Author \\ The Wealth of Networks (tinyurl.com/alrsa4j)
}

\begin{abstract}
How can small and medium-sized enterprises try open innovation and increase their level of collaboration with local partners? This article describes a possible solution: the Seeking Solutions approach. The Seeking Solutions process consists of four steps: a call for problems, problem selection, problem broadcast, and a collaborative event. This approach has been successfully used for the Quebec Seeks Solutions events in 2010 and 2012 with concrete results and real impacts. By mixing open innovation and collaboration, the Seeking Solutions approach has introduced a new concept: local open innovation.
\end{abstract}

\section{Introduction}

According to Geoffrey Nicholson, a former Vice President at 3M, "innovation is the transformation of knowledge into money" (tinyurl.com/38bfjeq). With such a definition, it is no surprise that innovation is a process that every company wants to master. Unfortunately, there is no magical recipe for innovation, only good practices.

Open innovation is increasingly recognized as one of the key practices to enhance the productivity of $R \& D$ and to improve an organization's capability for successful innovation. Several definitions of open innovation exist but I prefer the definition put forth by Frank Piller and colleagues (2010; tinyurl.com/ac7jqsh): "Open innovation is the formal discipline and practice of leveraging the discoveries of unobvious others as input for the innovation process through formal and informal relationships."

Piller's definition helps researchers and managers understand what open innovation is, but there still remains the question of how to do it. Although many large companies have established dedicated structures and methods for open innovation, few small and mediumsized organizations have truly embraced the open-innovation approach. There are three main reasons why "traditional" open innovation does not apply to small companies: i) they lack a means for enabling it - for example, they may lack knowledge about intellectualproperty management or cultural-change management to overcome the "not invented here" syndrome; ii) there are monetary barriers to try open innovation through intermediaries such as Innocentive (innocentive.com) or NineSigma (ninesigma.com) - the cost to post a problem with one of these companies is rarely below $\$ 30,000$; and iii) there are few open-innovation success stories in the current literature that relate to small or mediumsized organizations. To reinforce this point, Wim Vanhaverbeke (2012; tinyurl.com/ceq43m6) cautions that: "different rules apply and open innovation has to be reinvented to manage open innovation successfully in small companies."

Therefore, to reach small and medium-sized enterprises and to increase their capabilities to better innovate, one has to reinvent open innovation. I propose that a com- 


\section{Seeking Solutions: Solving Challenging Problems with Local Open Innovation}

\section{Christophe Deutsch}

bination of open innovation techniques and collaboration on a local basis is a solution that overcomes the key reasons why open innovation has not been widely adopted by small and medium-sized enterprises. The Seeking Solutions approach has been developed to realize this important combination of open innovation and collaboration at a local scale. Using a web-based broadcast technique, the approach encourages companies to diffuse a problem that they are not able to solve on their own. However, instead of ending the process with solutions proposed virtually - without any collaboration amongst the different solvers - a face-to-face working session is organized to stimulate collaboration and idea exchange around the problems that have been submitted. Seeking Solutions is neither just another openinnovation technique nor just another collaboration approach. It is a new mindset that combines both aspects to achieve local open innovation.

In this article, I first describe the genesis of the Seeking Solutions approach: the catalyst for the idea and the subsequent steps taken to refine it. Next, I describe the four steps of the methodology in detail: i) the call for problems, ii) problem selection, iii) problem broadcast, and iv) the collaborative event. I then report on concrete results following the first Quebec Seeks Solutions conference, which was held in 2010. Finally, I outline current and future development of the approach and provide conclusions.

\section{The Genesis of the Approach}

In 2009, I was Vice President of Operations at INO, an applied $R \& D$ centre in the province of Quebec, Canada. INO was part of the IDTEQ group (Regroupement pour l'innovation et le développement technologique de Québec; idteq.ca) with four other R\&D centres from the Quebec region. IDTEQ initiated a common project to increase collaboration amongst its members. The goal of the project was to build a database of available expertise as a starting point for future collaboration projects.

In June of that year, I participated in the International Society for Professional Innovation Management Conference (ISPIM; ispim.org) in Vienna, Austria, where I assisted the keynote presentation about open innovation given by Frank Piller (tinyurl.com/csy53hl). Although I was familiar with open innovation as a buzzword at many of the innovation conferences I had attended, this was the first time I truly appreciated what it was and what could be achieved with it. I found the concept both novel and exciting. When I returned to Quebec, I enthusiastically shared my thoughts with my colleagues from the IDTEQ project about this new way to innovate; they were quickly convinced that open innovation could help us collaborate more effectively, well beyond what we could hope to achieve through the expertise database we were then building.

From there, we attacked our collaboration project from another angle. First, we decided to prepare a training session on open innovation so that we would have a common understanding of the concept and be able to "speak the same language" of open innovation. In March 2010, we invited Frank Piller for a two-day working session, and around 80 people participated in the first training day. Attendees came from industry, research centres, academic institutions, and public sector organizations; they included researchers, engineers, technicians, managers, and representatives of municipal and provincial governments. The training was designed as an interactive learning experience, helping not only to deepen the understanding of open innovation but also offering also a new way to network.

For the second day, we limited the audience to the people from IDTEQ in order to prepare an action plan based on the following question: "From what we learned about open innovation, what should be done now?" The group was composed of people from different horizons - researchers, technicians, and managers - to ensure there would be a real output, not just a management decision. The main outcome of this session was a plan to organize a conference where people from the industry would present problems they face and that they are not able to solve themselves. Frank Piller found the idea interesting because most open-innovation initiatives typically arise from individual companies, whereas we were proposing a regional initiative. He challenged us to hold such an event before the end of 2010 , and we readily accepted his challenge.

In April 2010, we announced our intention to hold a "problem-solving conference" was announced on December 14th of that same year. A small group of champions from IDTEQ and Québec International (quebec international.ca), the economic development organization for the Quebec region organized this first event with support from consultants from Grisvert (grisvert.com), a company that specializes in the design, organization, and facilitation of collaborative events and organizational change. Later in the article, I will present concrete results from this first event, but in the next 


\section{Seeking Solutions: Solving Challenging Problems with Local Open Innovation}

\section{Christophe Deutsch}

section, I will first describe the local open innovation approach we developed for the event and refined through the initial conference and several other events that took place between 2010 and 2013.

\section{The Seeking Solutions Approach}

The Seeking Solutions approach to local open innovation consists of four major steps: i) the call for problems, ii) problem selection, iii) problem broadcast, and iv) the collaborative event. The four steps are illustrated in Figure 1, with further details provided in the subsections that follow.

\section{The call for problems}

The first step in the Seeking Solutions approach is the "call for problems", which is comparable to a call for papers in a traditional conference. However, in this case, the organizers are asking the wider community to submit challenging business problems that they have been unable to solve on their own. Sally Davenport and her co-authors in this issue (2012; timreview.ca/article/665) have described this type of call as "problemsourcing" because it is the inverse of crowdsourcing. With crowdsourcing, companies ask "a crowd" for solutions to a known problem; here, the crowd is being asked to put forth their problems, not their solutions.

The call for problems is an important step because the organizers of the conference have to convince people to submit a problem that can be put to the community. Common barriers for solution seekers include the "not invented here" syndrome (tinyurl.com/yuwk96) and inexperience with open innovation. Previous experience of open innovation, training, and testimonials from others can help solution seekers in the community overcome these barriers. If their problem is selected during the second step, the solution seekers will benefit from outside help working on their problem. However, the solution seeker will have to pay to participate to the event.

In our experience, a broadly cast call to a general "crowd" does not yield a sufficient quality or quantity of problems; a more targeted approach using the event organizer's network is often required. Even so, the organizers must carefully prepare the call for problems so that the advertisement is not perceived as spam, but as a real, value-added opportunity. A well-crafted call for problems will convince solution seekers that they cannot afford to miss this opportunity to try a novel approach to solving their important business problems.

The call for problems must last long enough to allow the advertisement to propagate and to give potential solution seekers time to consider and prepare their submissions. However, the call must be ended some time before the event to allow time for the next steps in the overall process. We recommend starting the call for problems at least six month prior to the event, and it should last for at least two months. These timelines can

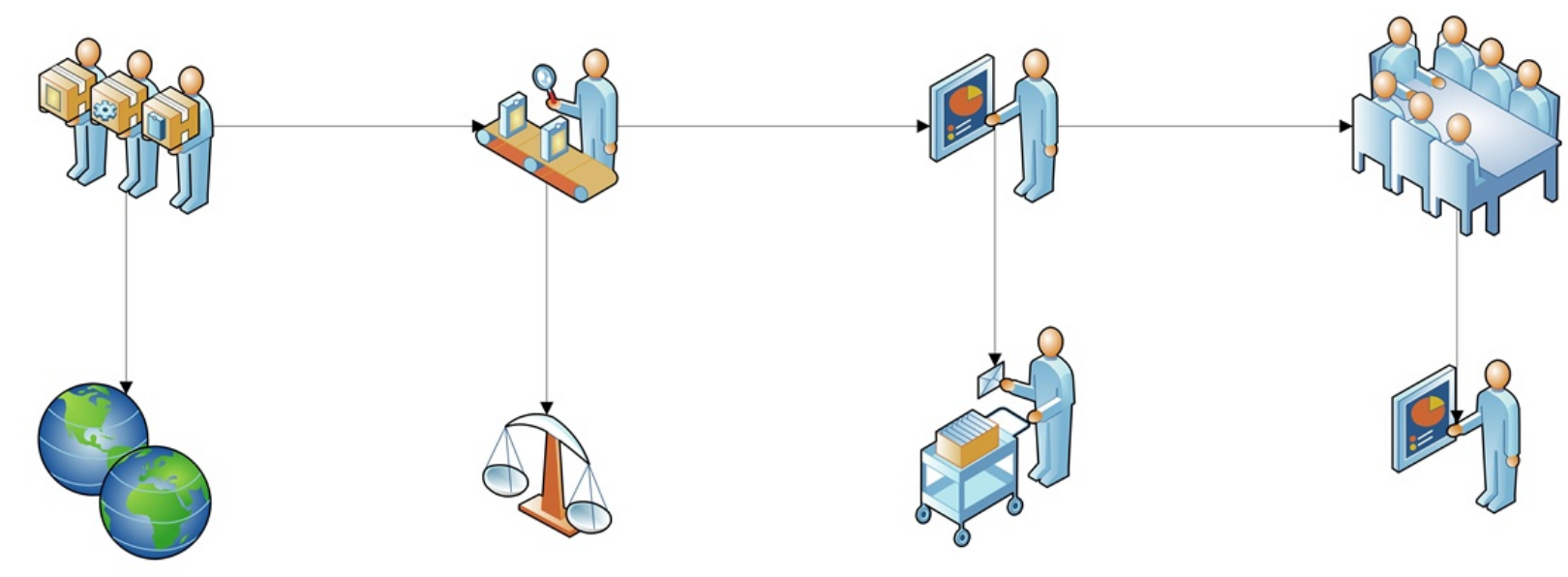

Step 1. The Call for Problems

- the problem-sourcing phase
Step 2. Problem Selection

- validation of the problems by Ambassadors
Step 3. Problem Broadcast

- through a dedicated web tool - both targeted and broad
Step 4. Seeking Solutions Event

- a real collaboration event

Figure 1. The four steps in the Seeking Solutions approach 


\section{Seeking Solutions: Solving Challenging Problems with Local Open Innovation}

\section{Christophe Deutsch}

be adjusted depending on the type of community. For example, if the event is aimed at an established industrial cluster where members are keen to try open innovation, a brief call for problems may be sufficient. At the end of this first step, a number of companies or institutions should have submitted some of their most critical and unsolved problems, and it will be up to the organizers to evaluate and select the most suitable of these problems.

\section{Problem selection}

The second step in the Seeking Solutions approach is "problem selection", which is required for two main reasons. First, the call for problems may have brought in more problems than could be addressed during a single event, for logistical reasons. Second, the selection process validates whether or not the problems submitted will really benefit from the approach. Just as not all types of problems can benefit from crowdsourcing (Piller and Wielens, 2012; tinyurl.com/bh7jq6n), not all types of problem can benefit from the Seeking Solutions approach. We use a common approach to validating problems that is used by NineSigma (ninesigma.com) and other open-innovation intermediaries. An expert of the problem's domain, called an ambassador in our case, is put in contact with the solution seeker. Just by asking some basic questions, the ambassador is able to help the solution seeker further define the problem and ensure that the description that will ultimately be posted on a web-based platform (in step 3 ) is sufficiently clear and broad.

Ambassadors play a key role in problem selection, and it is therefore important to identify potential ambassadors as early as possible when planning a Seeking Solutions event. An ambassador has to be a technical person with a good systemic view of the domain. They do not need to be an expert, but they have to be able to analyze a problem correctly and deeply. The ambassadors will not only help to define the problem but will also be the main point of contact for the solution seekers. Training in all aspects of the Seeking Solutions approach helps ambassadors guide the solution seekers throughout the process leading up to the event and during the event itself.

\section{Problem broadcast}

The third step in the Seeking Solutions approach is the "problem broadcast". The problems gathered and selected in steps 1 and 2 are broadcasted through a webbased platform including as much information as possible, such as figures, references, or details of failed solutions. The purpose of this step is to recruit potential problem solvers.
Two strategies are employed during the problem-broadcast step to reach potential problem solvers. First, the problems are broadcasted widely to reach a diverse range of potential problem solvers, without any preconceptions or constraints. A general broadcasting approach ensures that everyone who thinks they could help has the opportunity to participate in the collaboration event; this is an important, open-minded philosophy that allows solution seekers to gain all the potential benefits of open innovation. Links with local partners such as universities, $R \& D$ centres, and other members of the innovation community can help broadcast the problems wildly. Also, social media tools such as LinkedIn, Twitter, and Facebook have proven very efficient in broadcasting the problems to a large audience. Second, specialists are targeted based on expectations about what type of expertise might be relevant to a particular problem. In fact, we have seen that the solution seekers often already have an idea of who could help them; this information is used to target the broadcasting of problems to specific experts.

For the organizers of the event, this phase of the process is stressful because they do not fully know who will participate until a few days prior to the event. Will there be solvers for each of the submitted problems? Will there be enough solvers to make the event a success? In order to reduce this risk and complement the broadcasting strategy, different strategies can be applied, such as offering a reduced fee for students, inviting sponsors to pay for the participation of a specific group of people, or organizing a monetary reward for one of the problems. In our experience, such strategies were useful for initial events, but as more and more people become aware of the Seeking Solutions approach and the results it delivers, the less these strategies are required.

\section{The Seeking Solutions event}

The last step is what differentiates the Seeking Solutions approach from other open innovation techniques, because it involves a real event where non-virtual collaboration arises. Solution seekers and problem solvers come together during a full-day session to focus on the selected problems. This last step is crucial; careful preparation is required to ensure maximum output from the event. The process used for the event is simple and inspired by a framework described in the book Game Storming by Dave Gray and colleagues (2010; gogamestorm.com). The collaboration process is divided into three phases: i) divergence, ii) exploration, and iii) convergence. 


\section{Seeking Solutions: Solving Challenging Problems with Local Open Innovation}

Christophe Deutsch

The opening, or divergence, phase ensures that all the problem solvers understand the problems and that nothing important has been overlooked. Problem solvers are encouraged to ask questions so they fully understand the problems. This phase is significant and should not be rushed; although the event is just getting started, the foundations for later collaboration are being laid and potentially disruptive ideas may even arise at this point.

The second phase involves exploration and emergence: this is the moment where new ideas can arise and where the real collaboration happens. During this phase, the audience is guided with some generic questions from the facilitator, but each group can self-organize and use their time in their own way, depending on the progress being made. In this way, the exploration phase is an adaptation of the open-space technology introduced by Owen Harrison (2008; tinyurl.com/b7ppluw).

To end up with some concrete actions to solve the problems, the last phase helps people to converge. From the new ideas that have been submitted, the group decides which one is the best and how the solution seeker should act to validate it.

These three steps seem straightforward, but productive collaboration does not usually happen on its own; experienced facilitators are required. Facilitators can adapt the process in real-time, depending on the audience and on the progress made during the day. Grisvert (grisvert.com) has been an excellent partner for us in this regard.

Finally, to get the best out of this approach, it is also important to have a good environment. Figure 2 gives an example of a setting used during a Seeking Solutions event. Each solution seeker has their own "laboratory" where the participating problem solvers can engage in the collaboration process. In the laboratory, the solution-seeking company can display materials related to the problem, and computers are available to search the Internet or to sketch some initial ideas. The walls around the lab are used to capture the results of the different phases. The participants take notes at each of the tables in the laboratory and place them on the wall, leaving the solution seeker with the notes and ideas arising from the discussion of their problem.

The process is not designed to necessarily solve each problem within the timeframe of the event; rather, the goal is to explore each problem sufficiently to let new ideas emerge and to define concrete actions toward proposed solutions. We base our approach on Einstein's well-known quote: "If I had an hour to solve a problem, I'd spend 55 minutes thinking about the problem and 5 minutes thinking about solutions." The Seeking Solutions approach focuses on the 55 minutes spent thinking about the problem.
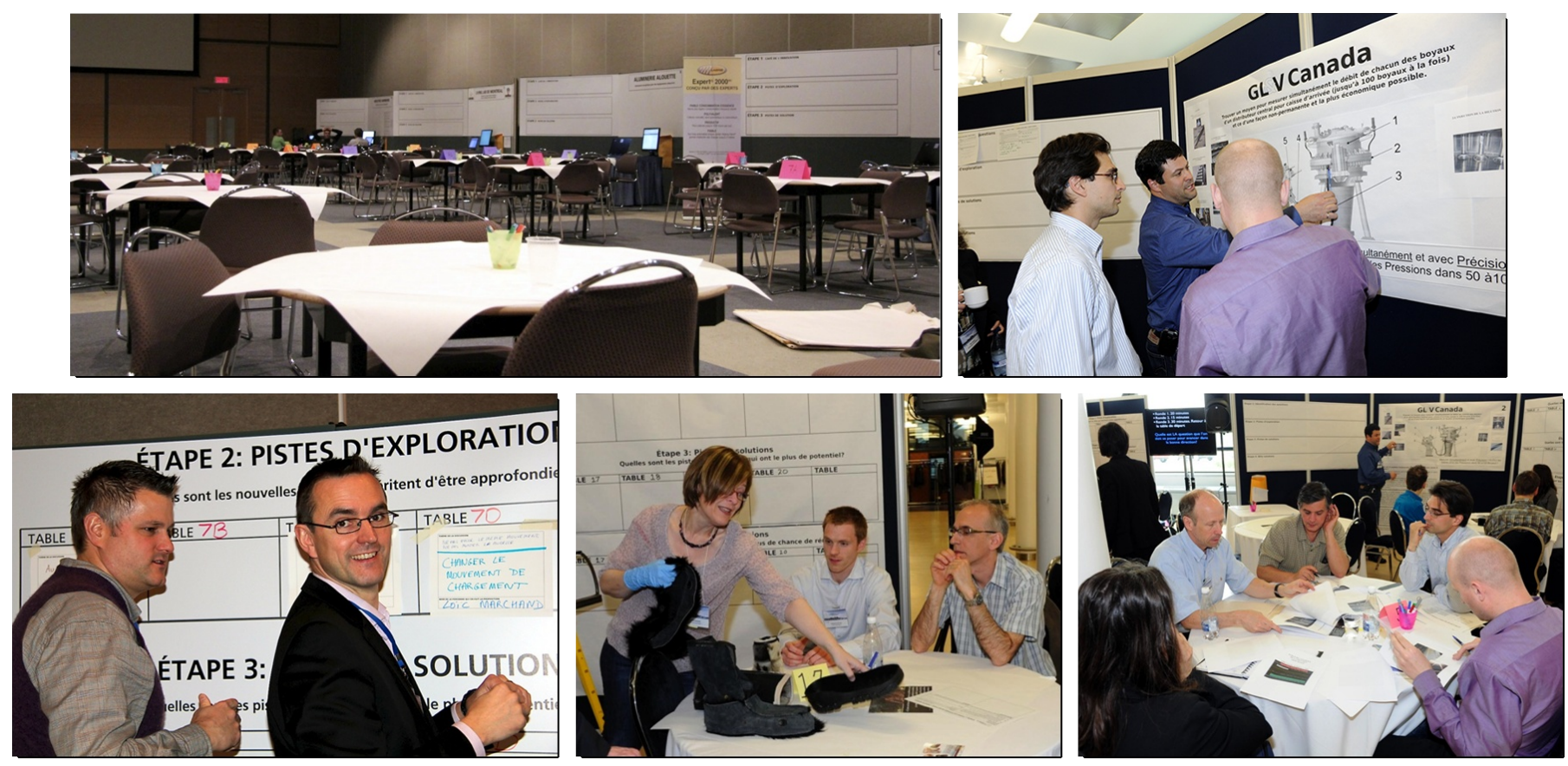

Figure 2. Solution seekers and problem solvers collaborating at a Seeking Solutions event 


\section{Seeking Solutions: Solving Challenging Problems with Local Open Innovation}

\section{Christophe Deutsch}

\section{The First Event: Quebec Seeks Solutions 2010}

The call for problems for the first event - Quebec Seeks Solutions (QSS) - was launched in June 2010. Ten problems were submitted by nine industrial companies from the Quebec area, despite the doubts and scepticism of many actors in the Quebec innovation ecosystem. The problems were broadcast online early in November 2010 thanks to the ambassadors. On the 14th of December 2010, 175 people gathered at the convention centre in Quebec City to attend the first problem-solving conference.

As mentioned earlier, solution-seeking companies were required to pay to participate, although the fee was set low to ensure participation and because the effectiveness of the approach had not yet been demonstrated. Notably, problem solvers were also required to pay to participate in the collaboration event. This fact astonished external analysts, who doubted whether anyone would be willing to pay to help solve companies' problems. However, there were two reasons for charging problem solvers. First, a fee ensures that everyone is committed to the process; it filters out those who would attend just "to see what's going on". Second, the fee reflects that there is significant value in participating in the event as a problem solver. In fact, demonstrating your capabilities to a potential customer in a real-life problem-solving context is probably the best way to do business development.

\section{Short-term feedback}

Immediately after the event, we interviewed a number of solution seekers and problem solvers to capture their direct feedback. All solution seekers confirmed that they gained a better understanding of their problems through the event. And, they either received new ideas they would not have found inside their companies or they confirmed that a pre-existing idea they were considering was the right one to pursue.

Both solution seekers and problem solvers rated the networking opportunity as the highest-value aspect of the event. One of the seekers told us that it would have taken weeks to meet as many interesting people if they had to contact them themselves and that they probably would not even have contacted some of them because they were outside their "traditional" network. Another seeker told us that it was incredibly valuable to meet people interested in the company's problems; they usually only encounter people who are pre-occupied with selling their own products or services to the company.
Finally, on the problem solver's side, participants appreciated the opportunity to prove their value to the companies. For example, one consultant had been trying (unsuccessfully) to get an appointment with one of the solution-seeking companies; after he had actively and constructively participated during the event to solve the company's problem, the R\&D manager of this company asked the consultant for a meeting.

Observers were surprised that no intellectual-property issues were raised during the event. Prior to the event, we had clearly stated that all the discussions would be "open source" and each participant had to sign a disclaimer when they registered. Our major sponsor, Fasken Martineau (fasken.com), supported us in kind by offering to all the participants the support of two intellectual-property lawyers to answer any questions that arose during the event. However, no such questions stopped any of the discussions or restricted the emergence of new ideas. It was only after the event - when companies began implementing the solutions - that questions about intellectual property arose. With hindsight, we realize that this was the right time for intellectual-property issues to come up. The event itself is an exploration of the problem where everybody feels free to contribute; intellectual-property challenges typically arise "downstream" from this initial exploration. The reason that intellectual-property issues arise later in the process is likely related to the complexity of the problems and their potential solutions. When a promising idea on how to solve a problem is submitted, the intellectual property required to implement the idea might not be straightforward; in many cases, it is more efficient for the solution seeker to work with the solver that came up with the idea than to try to implement the idea on their own.

Finally, due to the open-innovation aspect of the Seeking Solutions approach, we were expecting that some unobvious connections would happen. Take the example of Kruger, a paper mill company that submitted the following problem: they needed to find innovative applications to use their new coating machine in their Trois Rivières' plant or else they would be forced to close the plant. Natural problem solvers would have been found within the paper industry, but the innovative solution actually came from a researcher in the agro-environmental domain who proposed that Kruger should produce paper tarpaulins with embedded fertilizer to preserve soil humidity and fertilize the crops. This solution was unobvious and highlighted the importance of "outsider" input into the innovation process. 


\section{Seeking Solutions: Solving Challenging Problems with Local Open Innovation}

\section{Christophe Deutsch}

\section{Direct impacts one year later}

We decided to follow up with the solution seekers to validate that the enthusiasm of the direct feedback after the event was not only a short-term result. When we contacted the companies 10 months after the event to measure the real impact of the event, we found the following results:

1. Sixty per cent of the solution-seeking companies stayed in contact with people they met during the event. For example, one company developed a sustained relationship a research centre and two technology transfer centres following on from their interactions during the event.

2. The largest perceived impact for all the companies remained the value-added networking.

3. Real business opportunities appeared, and some companies did award contracts to problem solvers that proposed good ideas during the event. The most significant result is the Kruger case mentioned earlier. Two $R \& D$ centres proposed a feasibility study to Kruger, developed a tarpaulin, and began testing it less than eight months after the event. Following this preliminary feasibility study, these two R\&D centres, along with Kruger and other industrial partners, proposed a half-milliondollar joint project that has been funded by the Province of Quebec.

4. The fourth impact was less concrete, but is just as important. It involved a change in culture or mindset among the participants. Some companies increased their level of openness as a result of some of their staff experiencing this open innovation approach. One solution-seeking company told us that they did not solve their problem directly during the event, but six months later, when facing a new problem, the team said: "Shouldn't we apply what we learned from the Seeking Solutions approach and try to see if there's not a solution outside our domain?" This question stimulated an Internet search for similar problems, and they discovered that the pharmaceutical industry had experienced exactly the same type of problem and had solved it. An inexpensive product existed already on the market and they bought it.

\section{Other Events and Next Steps}

A second edition of Quebec Seeks Solutions took place in May 2012. Nine solution-seeking companies and 162 problem solvers participated in the two-day event. We are in the process of analyzing the results gathered 10 months after that event; the initial feedback suggests that the concrete results will be equivalent to the 2010 event.

We also conducted a workshop called ISPIM Seeks Solutions during a June 2012 conference in Barcelona, Spain. The process was slightly adapted to the duration of the event and the context of the conference. The workshop lasted less than two hours and the call for problems was targeted only to innovation management problems. Thirteen 13 problems were submitted and five were selected for the workshop. The solution seekers appreciated the experience even though it only gave them a preview of what could be achieved in a fullday event.

Thanks to the success of the first editions of Quebec Seeks Solutions, and thanks to the support of contributors such as Quebec International (quebecinternational.ca) and IDTEQ (idteq.ca), I co-founded a new startup company called En Mode Solutions (enmodesolutions.com) in fall 2012. En Mode Solutions promotes the Seeking Solutions approach and offers its services to help companies, conferences organizers, industrial consortia, cities, and economic development organizations to hold Seeking Solutions events all around the world.

In February 2013, we conducted a similar workshop with the Technology Innovation Management (TIM; carleton.ca/tim) program at Carleton University in Ottawa, Canada; a summary of this event is provided later in this issue of the TIM Review (timreview.ca/article/669).

Several other events are already planned for 2013.The third edition of Quebec Seeks Solutions will take place in November. "Polymères en mode solutions" is an event for the Quebec Composite Industrial Consortium (tinyurl.com/be62b38) and will take place in September in Saint-Jean-sur-Richelieu, near Montreal, Quebec. The second edition of the ISPIM Seeks Solutions workshop will be held in Helsinki in June around innovation management problems, and we encourage you to submit problems here: tinyurl.com/9whhvhs

The growing interest in the Seeking Solutions approach confirms that local open innovation holds appeal to companies looking for innovative solutions to their challenging problems. 


\section{Seeking Solutions: Solving Challenging Problems with Local Open Innovation}

\section{Christophe Deutsch}

\section{Conclusion}

The Seeking Solutions approach, as developed through the Quebec Seeks Solutions events, has introduced a new concept: local open innovation. This new concept is a combination of open innovation and collaboration on a local scale. This approach brings new ways to solve problems, to network, to create business opportunities, and to innovate. Small companies tried the approach and received real benefits from it, thereby demonstrating that the Seeking Solutions approach has reinvented open innovation so that it is now accessible to small and medium-sized enterprises.

Aside from the contexts described in this article, the approach can be used to encourage open innovation with large companies, inside local consortia, as a conference workshop, or simply to bring a new dynamic within a region.

Many problems that company faces today are not only complicated they are complex, often mixing technical, environmental, social, and political aspects. To face this growing complexity, classical problem-solving methodologies are no longer appropriate. The Seeking Solutions approach has the potential to address our complex challenges, and we believe that it can help us to migrate from a collection of intelligences to a real collective intelligence. The next step is to encourage more and more companies to try local open innovation.

\begin{abstract}
About the Author
Christophe Deutsch is $R \& D$ Manager at Telops (telops.com), an innovative company in the field of high-performance infrared sensors. He is responsible for the successful realization of product development and R\&D projects. Previously, he was Vice President Operations at INO, an applied R\&D centre in the field of optics, where he implemented projectmanagement and technology-development processes and co-founded the RCR, a circle of $R \& D$ managers. Christophe has also worked for ABB Analytical Solutions, where he developed his competencies in system engineering and project management in several aerospace projects. As a member of ISPIM's advisory board, he promotes innovation management to increase efficiency of R\&D. In 2012, he co-founded En Mode Solutions (enmodesolutions.com).
\end{abstract}

Citation: Deutsch, C. 2013. The Seeking Solutions Approach: Solving Challenging Business Problems with Local Open Innovation. Technology Innovation

Management Review. March 2013: 6-13.

Keywords: local open innovation, open innovation, crowdsourcing, broadcast of search, collaboration, Seeking Solutions 